\title{
A Quality Improvement Intervention to Inform Scale-Up of Integrated HIV-TB Services: Lessons Learned From KwaZulu-Natal, South Africa
}

\author{
Santhanalakshmi Gengiah, ${ }^{a}$ Kogieleum Naidoo, ${ }^{a, b}$ Regina Mlobeli, ${ }^{a}$ Maureen F. Tshabalala, ${ }^{c}$ \\ Andrew J. Nunn, ${ }^{d}$ Nesri Padayatchi, ${ }^{a, b}$ Nonhlanhla Yende-Zuma, ${ }^{a, b}$ Myra Taylor, ${ }^{,}$Pierre M. Barker, ${ }^{c, f}$ \\ Marian Loveday ${ }^{\text {,g }}$
}

\section{Key Findings}

- The quality improvement (QI) intervention was able to guide clinic staff in developing simple but effective change interventions, using resources already available, to improve HIV-TB integrated service delivery.

- The QI intervention implemented dramatically improved isoniazid preventive therapy initiation rates among eligible HIV patients and resulted in moderate improvements in HIV testing and screening, TB screening, and viral load monitoring.

\section{Key Implications}

- Program managers should ensure that all categories of health care workers from all levels of the health care system are included in QI workshops or learning sessions to harness the knowledge and experiences of all role players working within the system.

- QI implementers should consider adopting a combination approach to improvement interventions, such as QI training combined with mentorship, collaborative learning, and data QI activities.

- To strengthen and ensure the success of QI interventions, senior-level program managers should consider allocating resources (human, financial, and infrastructure) dedicated to data QI for a sustainable and effective QI program.

\footnotetext{
${ }^{a}$ Centre for the AIDS Programme of Research in South Africa (CAPRISA), Durban, South Africa.

${ }^{b}$ Medical Research Council-CAPRISA HIV-TB Pathogenesis and Treatment Research Unit, Doris Duke Medical Research Institute, University of KwaZuluNatal, Durban, South Africa.

${ }^{c}$ Institute for Healthcare Improvement, Cambridge, MA, USA.

${ }^{\mathrm{d}}$ Medical Research Council, Clinical Trials Unit at University College London, London, United Kingdom.

e School of Nursing and Public Health, University of KwaZulu-Natal, Durban, South Africa.

fDepartment of Maternal and Child Health, Gillings School of Global Public Health, University of North Carolina, Chapel Hill, NC, USA.

gHIV Prevention Research Unit, South African Medical Research Council, South Africa.

Correspondence to Santhanalakshmi Gengiah (Gengiahs@gmail.com).
}

\section{ABSTRACT}

Introduction: In South Africa, mortality rates among HIV-TB coinfected patients are among the highest in the world. The key to reducing mortality is integrating HIV-TB services, however, a generalizable implementation method and package of tested change ideas to guide the scale-up of integrated HIV-TB services are unavailable. We describe the implementation of a quality improvement (QI) intervention, health systems' weaknesses, change ideas, and lessons learned in improving integrated HIV-TB senvices. Methods: Between December 1, 2016, and December 31, 2018, 8 nurse supervisors overseeing 20 primary health care (PHC) clinics formed a learning collaborative to improve a set of HIVTB process indicators. HIV-TB process indicators comprised: HIV testing services (HTS), TB screening among PHC clinic attendees, isoniazid preventive therapy (IPT) for eligible HIV patients, antiretroviral therapy (ART) for HIV-TB coinfected patients, and viral load (VL) testing at month 12. Routine HIV-TB process data were collected and analyzed.

Results: Key change interventions, generated by health care workers, included: patient-flow redesign, daily data quality checks; prior identification of patients eligible for IPT and VL testing. Between baseline and post-QI intervention, IPT initiation rates increased from $15.9 \%$ to $76.4 \%(P=.019)$, HTS increased from $84.8 \%$ to $94.5 \%(P=.110)$, TB screening increased from $76.2 \%$ to $85.2 \%(P=.040)$, and $V L$ testing increased from $61.4 \%$ to $74.0 \%(P=.045)$. ART initiation decreased from $95.8 \%$ to 94.1\% $(P=.481)$.

Discussion: Although integrating HIV-TB senvices is standard guidance, existing process gaps to achieve integration can be closed using Ql methods. QI interventions can rapidly improve the performance of processes, particularly if baseline performance is low. Improving data quality enhances the success of $\mathrm{Q}$ initiatives.

\section{INTRODUCTION}

n South Africa, TB remains a public health challenge largely driven by a high background prevalence of HIV, estimated at $12 \%$ in the general population. ${ }^{1}$ In 2019 , an estimated 58,000 people died from TB, of whom $36,000(62 \%)$ were coinfected with HIV. ${ }^{2}$ For South Africa to achieve its goal of reducing TB deaths by $95 \%$ by 2035 , steps to accelerate the reduction in $\mathrm{TB}$ mortality are needed, specifically in HIV-TB coinfected patients. ${ }^{3}$ 
Integrating HIV and TB services (hereafter written HIV-TB services) is a key strategy in reducing TB-related deaths among people living with HIV. ${ }^{4}$ HIV-TB services refers to screening, diagnosis, and treatment services provided for both diseases at the same clinic, by the same clinic team, on the same visit day. ${ }^{5,6}$ We have previously published the key evidence-based, clinical HIV-TB integration activities that have been shown to reduce $\mathrm{TB}$-related mortality among people with HIV, TB, and both HIV and TB. ${ }^{7}$ Specific integration services include HIV testing services (HTS) for all TB patients, TB screening for all clinic attendees, isoniazid preventive therapy (IPT) initiation for eligible HIV patients, antiretroviral therapy (ART) and cotrimoxazole for all HIV-TB coinfected patients, and retention and treatment adherence monitoring. ${ }^{7}$ All HIV-TB integration activities mentioned are incorporated into the South Africa National Department of Health (DOH) HIV treatment guideline document. ${ }^{8}$ However, suboptimal implementation of HIV-TB services in public health facilities has been observed where opportunities to screen patients for TB, test for HIV, and subsequent linkage to treatment have been missed. ${ }^{3,9,10}$ While patientrelated factors such as stigma and fear of HIV testing may be contributing to gaps in the HIV-TB care cascade, there is mounting concern that weaknesses in health care systems at the frontline are not adequately addressed. ${ }^{11}$

Operationalizing and delivering high-quality HIV-TB services is complex and challenging in resource-constrained settings. ${ }^{5,12}$ The need for simple, low-cost, and sustainable solutions to enhance service delivery was the impetus for introducing quality improvement (QI) methods in public health settings. ${ }^{13,14}$ The defining principle of QI is the focus on improving underlying health systems and addressing gaps with feasible solutions. ${ }^{15}$ In South Africa, QI was successfully implemented to reduce mortality in mothers, neonates, and infants. ${ }^{16,17}$ However, little is known of the effectiveness of QI in reducing mortality in patients accessing public health facilities for HIV, TB, and HIV-TB services. ${ }^{7}$

The Centre for the AIDS Programme of Research in South Africa (CAPRISA), implemented a cluster-randomized trial, the scaling up TB and HIV treatment integration (SUTHI) trial, designed to test the effectiveness of a QI intervention in enhancing HIV-TB service integration to reduce mortality in HIV-TB patients. ${ }^{7}$ CAPRISA, in partnership with the Institute for Healthcare Improvement (IHI), designed and implemented a
QI intervention to enhance HIV-TB service delivery by identifying and addressing the health system's weaknesses at the primary health care (PHC) clinic level. ${ }^{7}$

In this article, we describe the QI intervention, our theory of change, report the impact of the intervention on HIV-TB services, identify changes that were associated with improved processes outcomes, and elucidate challenges associated with implementing QI to improve HIV-TB services in PHC clinics.

\section{METHODS}

\section{The SUTHI Trial}

The design and rationale for the SUTHI trial are published elsewhere. ${ }^{7}$ Briefly, SUTHI was a cluster-randomized trial in which 16 PHC nurse supervisors (clusters) and the 40 PHC clinics under their oversight were randomly assigned to receive either a structured program of QI training and mentorship to expand the skill and capacity of health care workers in improving HIV-TB services (QI intervention group) or to the standard of care supervision and support (SOC) group as carried out by the South Africa DOH. Eight nurse supervisors and their 20 clinics were assigned to the QI intervention group and 8 nurse supervisors and their 20 clinics were assigned to the SOC group. All clinics were followed up for 18 months.

\section{Setting}

The SUTHI trial was located in the King Cetshwayo District and Ugu District in KwaZulu-Natal Province, South Africa. The King Cetshwayo District and Ugu Districts have reported incident TB rates of 859 per 100,000 and 810 per 100,000 , respectively; antenatal HIV prevalence rates of $33.4 \%$ and $41.7 \%$, respectively; and mortality rates attributable to TB and HIV of $36 \%$ and $35 \%$, respectively. ${ }^{18}$ Given the high rates of $\mathrm{TB}$ and HIV, both districts were ideal locations for the SUTHI trial. In South Africa, PHC clinics are the first point of entry into the health care system for a large majority of the population and services are free. ${ }^{7}$ The South African DOH HIV treatment guidelines recommends provision of integrated HIV-TB health care as standard practice. ${ }^{8}$

\section{Standard Support and Supervision}

The QI intervention in the SUTHI trial was implemented in parallel to other improvement activities undertaken by the district management team (DMT). Improvement initiatives undertaken by the DMTs were considered as a SOC and were
A QI intervention was implemented to enhance HIV-TB service delivery by identifying and addressing the health system's weaknesses at the primary health care clinic level. 
available to the QI group clinics and the SOC group clinics. Both study districts were supported by a highly motivated DMT who conducted routine, in-person, quarterly PHC clinic visits, and weekly data-driven progress update meetings with representatives from all facilities, including SUTHI study clinics. DMT involvement was maintained throughout the study period. Support from local, nongovernmental organizations (NGOs) for the improvement of the HIV and TB programs in both districts were present both before and during the study.

\section{CHANGE THEORY}

A change package to guide implementation of HIV-TB services was not available. Instead, we implemented an intervention that would allow change ideas to emerge from the input and experiences of the clinic staff and nurse supervisors in the QI intervention group. Our change theory was premised on a collective understanding from published articles and feedback from implementers on the primary and secondary drivers of poor performance in HIV-TB service integration (Figure 1). Primary elements of our change theory were:
(1) HIV-TB clinical content comprising a package of essential evidence-based interventions supported by an implementation algorithm suitable for a clinic setting, (2) implementation content comprising health care worker training and clinical skills capacity building for improved identification and treatment of HIV-TB patients as well as training in QI methodology; and (3) data quality improvement to enhance reliability and completeness of routine HIV-TB data.

\section{Clinical Content}

The development of the package of HIV-TB services was preceded by a review of published literature, South African HIV and TB treatment guidelines and policies, and input from experts in the field of HIV-TB co-management to identify the most effective evidence-based clinical activities associated with a reduction in mortality in HIV-TB coinfected patients. We assembled key HIV-TB clinical services into an HIV-TB care algorithm (Figure 2) that served as a training tool for QI group clinics. Health care workers in the QI collaborative were trained to appropriately identify, triage, and treat HIV-TB patients and prevent TB in HIV patients.

FIGURE 1. Change Theory Based on Primary and Secondary Drivers of Poor Performance in Integrated HIV-TB Services and Change Concepts Used in a Quality Improvement Intervention for HIV/TB Service Integration in KwaZulu-Natal, South Africa

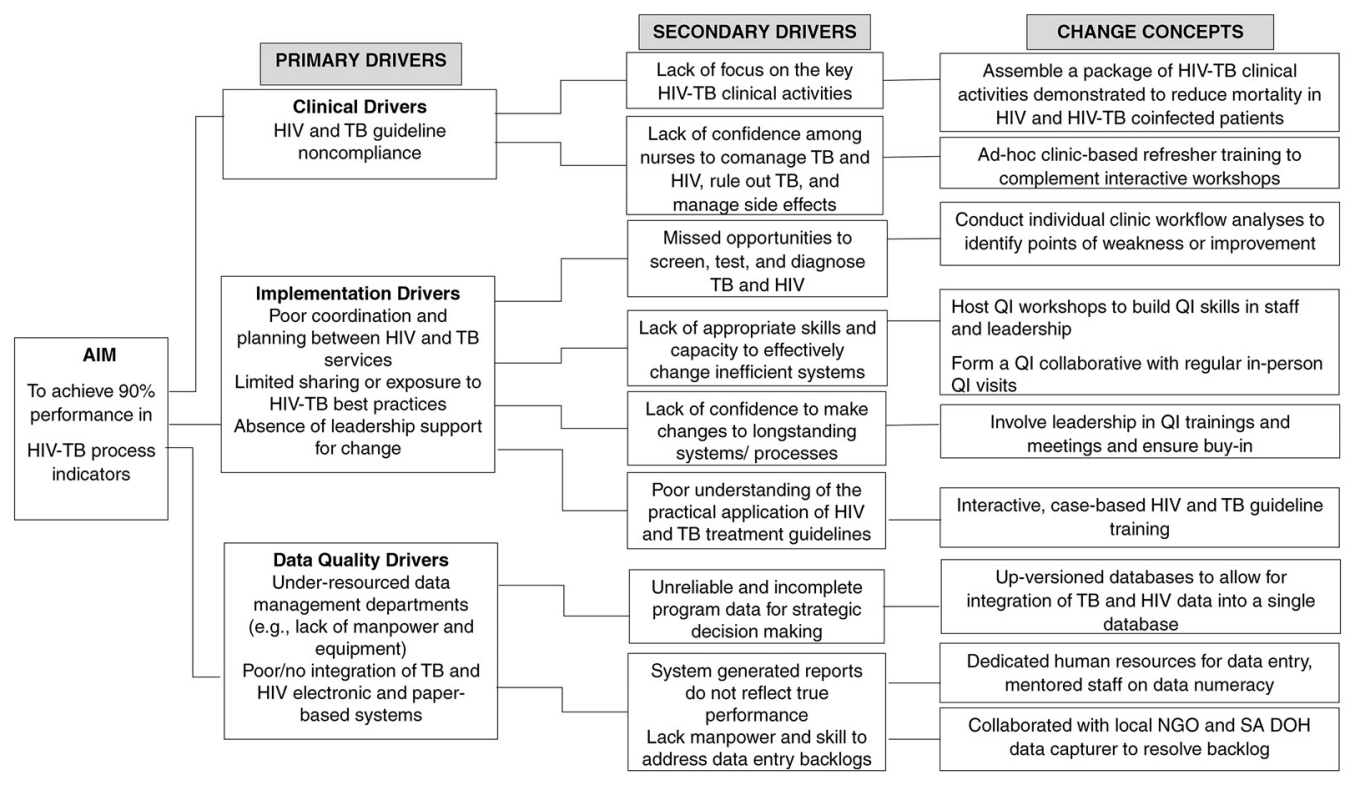

Abbreviations: NGO, nongovernmental organization; QI, quality improvement; SA DOH, South African Department of Health. 
FIGURE 2. Key HIV-TB Services Care Algorithm Training Tool Used in a Quality Improvement Intervention for HIV/TB Service Integration in KwaZulu-Natal, South Africa

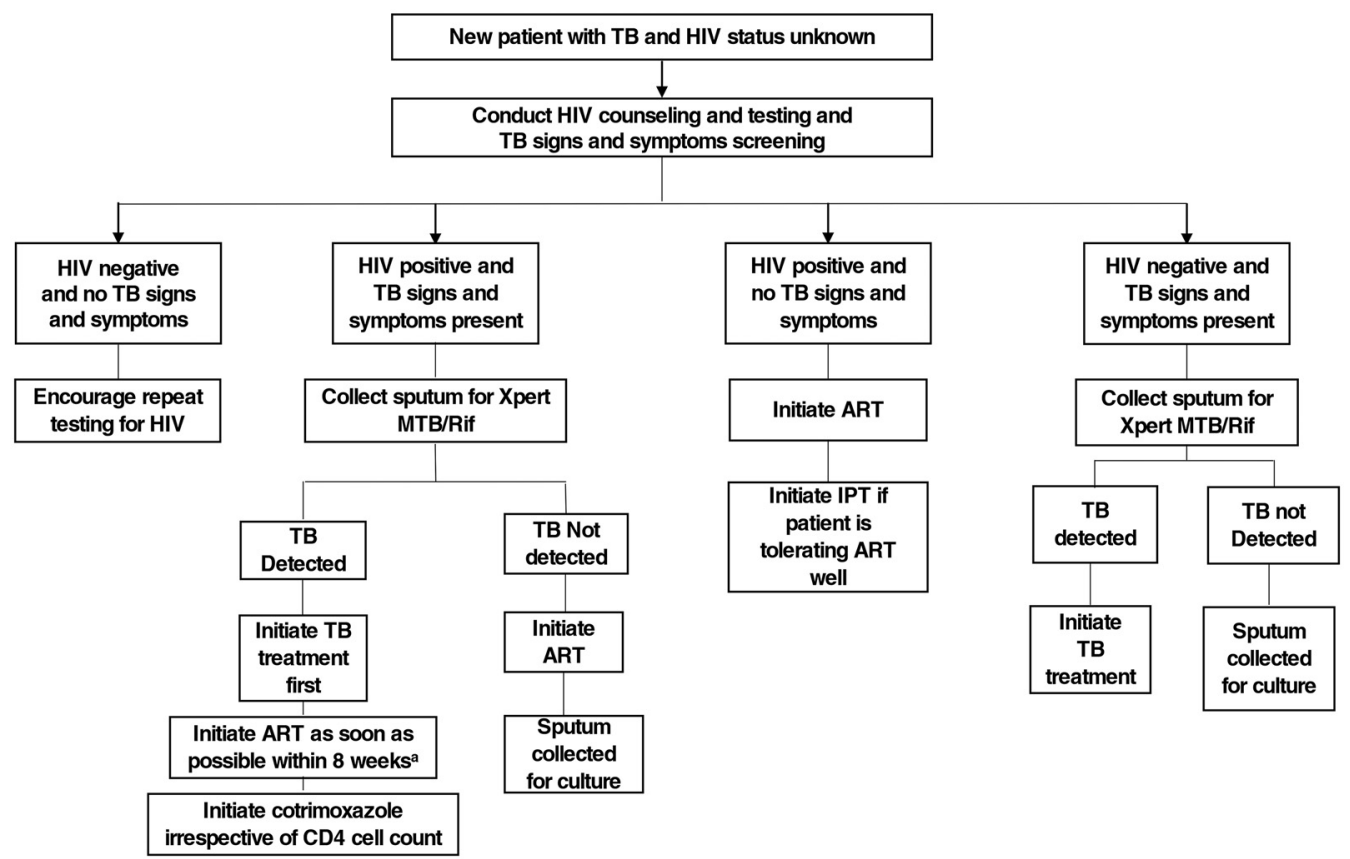

Abbreviations: ART, antiretroviral therapy; IPT, isoniazid preventive therapy; Xpert/MTB/Rif, a rapid, molecular, cartridge-based test used for TB diagnostics that provides an immediate rifampicin resistance result.

a For HIV-TB coinfected patients: If CD $4<50$ cells $/ \mu$, initiate ART within 2 weeks of starting TB treatment AND if CD4 $>50$ cells $/ \mu$, initiate ART within 2-8 weeks of starting TB treatment.

\section{Implementation Content}

Historically, HIV and TB services operated separately; however, the directives, policies, and guidelines from South Africa National DOH to colocate and integrate both services at a single facility, without adequate implementation guidance, failed to integrate HIV-TB health care delivery. ${ }^{5}$ Efficient integration of services requires joint planning and coordination between different departments within a clinic together with the provision of relevant training. ${ }^{19}$ We undertook to ensure that staff had the clinical skills to find and treat HIV-TB coinfection and quality improvement skills to strengthen and optimize HIV-TB patient flow and workflow processes.

\section{Improving Clinical Skills in Screening, Diagnosis, and Management of HIV-TB Coinfection}

At the start of the study, a l-day training workshop in each district was conducted for the QI collaborative with a study-appointed clinician trainer and members of the DMT serving as facilitators.
The training session emphasized that integrated HIV-TB services meant delivering both HIV and TB care and treatment at the same facility, by the same clinic team on the same day, also known as "the single facility approach." Training content included a review of the Xpert MTB/RIF algorithm for the screening and diagnosis of $\mathrm{TB}$; timing and criteria for ART initiation in TB patients; HIV-TB comanagement in adults, pregnant women, and pediatrics; and utilization of data reports from routine electronic databases to track health systems performance. An interactive, case-based mode of teaching was adopted where treatment and patient scenarios resembled typical real-world situations to which the audience could relate.

\section{Use of QI Methods to Improve Integrated HIV-TB Services}

In this study, we used the Model for Improvement as the methodological framework to identify systems' weaknesses and optimize workflow to enhance the performance of HIV-TB services to 
acceptable standards stipulated in the UNAIDS 9090-90 strategy document (Supplement Figure 1). ${ }^{20}$

Each clinic in the QI collaborative reviewed their clinic patient flow to understand the system and identify weaknesses, bottlenecks, or potential improvements that would strengthen HIV-TB care delivery. The clinic QI team consisted of 1 representative from each staff category to ensure all perspectives were represented. Whenever possible, PHC clinic supervisors and clinic operations managers participated in biweekly QI meetings.

The plan-do-study-act (PDSA) cycle was the guiding framework used to test and accumulate knowledge on proposed change ideas. During the plan phase, appropriate clinic team members, who would test the change idea, were identified and roles and responsibilities explained. Change ideas were recorded and as a QI team member tested changes (do phase), other team members collected process data and made observations of any unintended or negative impacts on the system. During the study phase, annotated run charts were used to track the performance of HIV-TB service outcomes and reviewed every 2 weeks by QI clinic teams. In the act phase, the QI team decided on adapting, adopting, or abandoning change ideas. On average, 4 PDSA cycles per HIV-TB indicator were completed before a change idea was perfected and adopted.

\section{By partnering with IHI, the SUTHI trial gained an experienced leader in QI implementation methods.}

and thereafter reduced to monthly face-to-face visits in the last 6 months of the study. Face-to-face visits included meeting with the clinic QI teams, observing the clinic teams in their daily routine, and ensuring implementation of QI plans.

\section{Improving Data Quality}

A roving team of study-appointed data capturers conducted regular quality assurance checks on patient registers, chart notes, and electronic HIV and TB databases maintained at the clinic. Paper-based systems were checked for completeness, legibility, and accuracy. Every 6 months patient chart note data were compared to the electronic system data for a randomly selected sample of HIV, TB, and HIV-TB patients. Feedback on discrepancies, incorrect, or missing data was given to clinic teams. The roving team assisted with clearing major backlogs in data entry.

\section{Key Inputs for QI Intervention Implementation}

The implementation of the QI intervention required the establishment of a partnership between CAPRISA and IHI, appropriately skilled staff to drive the QI activities, and technically skilled data staff to improve data quality.

Local QI expertise, with formal QI training and practical experience, was a scarce resource at the start of the trial. By partnering with IHI, the SUTHI trial gained an experienced leader in QI implementation methods. At the design phase of the study, IHI played a key role in training study staff in QI methods using a train the trainer model. Two study-appointed professional nurses (1 per study district) trained by IHI, drove the QI process at the clinic level and were under the oversight of a QI advisor from IHI who provided mainly virtual support. Each nurse supported 10 QI clinics. Between study enrollment to month 12 made fortnightly, the nurse made in-person mentorship visits to QI clinics. These visits were reduced to monthly mentorship visits between month 13 and month 18.

A data manager based at the CAPRISA headquarters oversaw the roving data quality improvement team that consisted of 2 data coordinators ( 1 per district), and 6 data capturers ( 3 per district). The intervention was implemented in the context of a cluster-randomized trial and to ensure that we had comparable data in the QI clinics and SOC clinics, the data team conducted improvement activities in both study groups during the 
study. The data team made fortnightly visits to QI improvement clinics and similarly to SOC clinics.

In addition, due to the nature of the trial design, learning sessions were held in conference venues and not on South Africa DOH premises. All costs of the venues, accommodation for trainers, and transport of health care workers were borne by the study.

\section{Study Outcomes and Data Collection}

HIV-TB process indicators were collected every month from paper-based registers (ART, TB, and HIV registers), electronic databases, and patient chart notes. These data were recorded onto paperbased data collection tools and faxed to the central office. Training registers were completed at each QI workshop, recording the number and designation of health care workers that attended. The QI nurse mentor and clinic QI team maintained detailed records on a PDSA template (provided by IHI) of the dates that QI work began per indicator and the change ideas, adaptations, and challenges encountered. The completed PDSA templates were submitted for analysis. Table 1 defines the HIV-TB process indicators that clinic teams selected for improvement and data elements used to calculate performance. For ease of reference, a shortened name (abbreviation) was assigned to each indicator in Table 1 and will hereafter be used in all subsequent sections.

\section{Statistical Analysis}

We analyzed data at the nurse supervisor level (the cluster). Monthly performance for each HIVTB process outcome was calculated by summation of numerators of all clinics that comprised a cluster and divided by the sum of the denominators of all respective clinics in the cluster. The mean of all cluster means reflected the monthly performance, which was then plotted as $x m r$-charts (Figure 3). A run of 8 or more data points on 1 side of the center line was defined as a shift and a run of 8 or more data points in an upward or downward direction was defined as a trend. ${ }^{22}$ Geometric means were calculated as a single estimate of baseline performance (last 6 months before study enrollment) and for the post-QI intervention phase (months 13-18) (Table 2). The absolute difference between the post-QI intervention geometric mean and the baseline geometric mean was calculated to reflect the size and direction of the improvement. Paired $t$-test was used to determine if differences between

TABLE 1. Definitions of HIV-TB Process Indicators Used in the Quality Improvement Intervention to Integrate HIV-TB Services in KwaZulu-Natal, South Africa

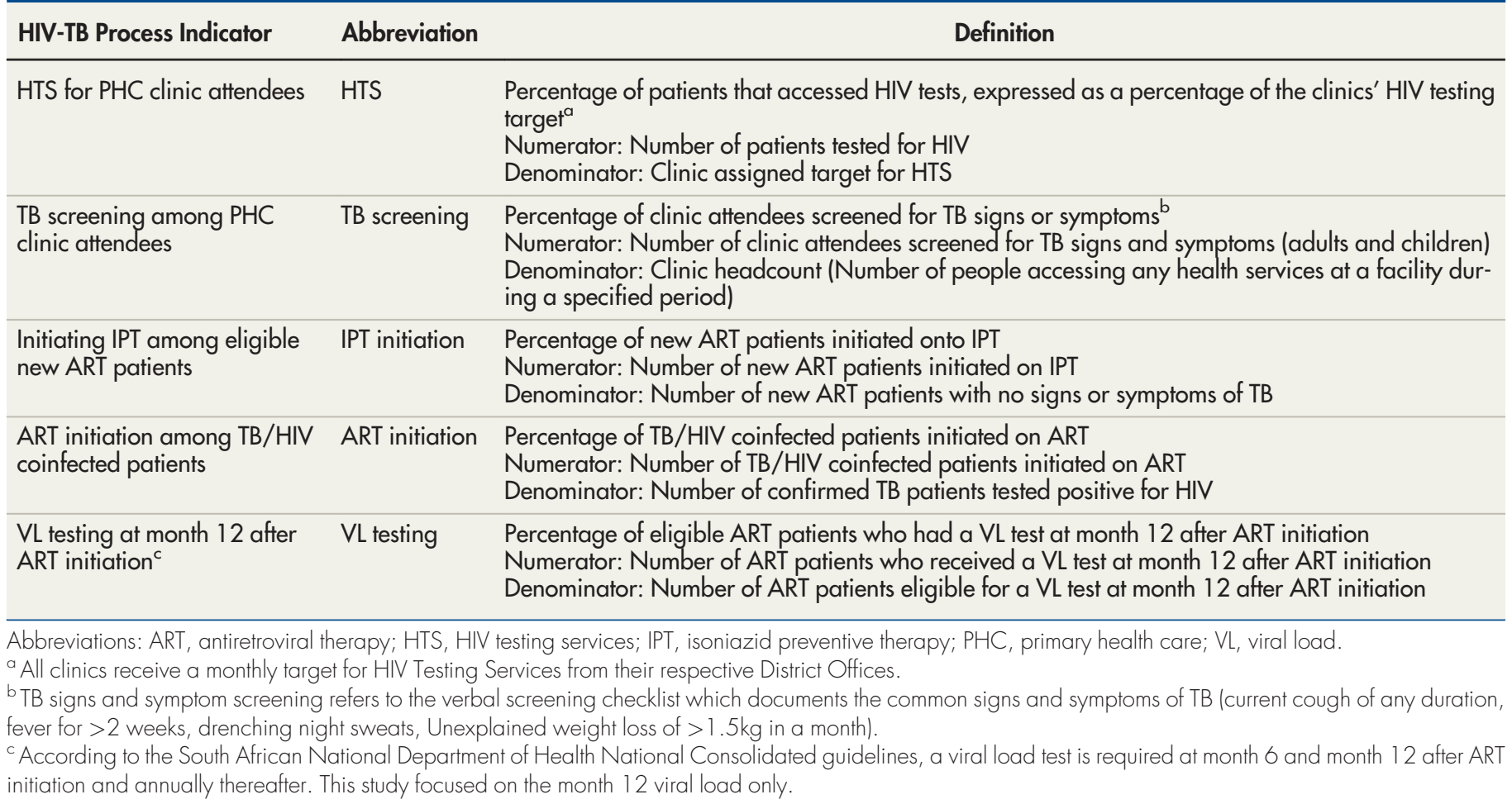


TABLE 2. Summary of Changes in HIV-TB Process Indicators Used in the Quality Improvement Intervention to Integrate HIV-TB Services in KwaZulu-Natal, South Africa

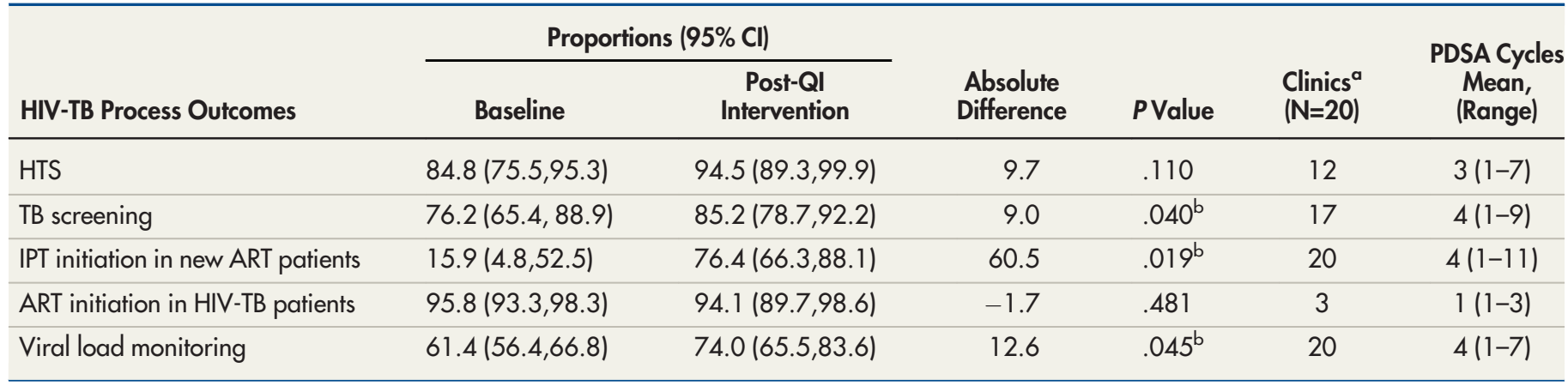

Abbreviations: ART, antiretroviral therapy; Cl, confidence interval; HTS, HIV testing services; IPT, isoniazid preventive therapy; PDSA, plan-do-study-act; QI, quality improvement.

a Number of clinics engaged in quality improvement.

b $P$ value significant at $<.05$ using paired ttests.

FIGURE 3. xmr Charts of Monthly Performance in HIV-TB Process Indicators in a Quality Improvement Intervention for HIV/TB Service Integration in KwaZulu-Natal, South Africa (a) Percentage of Eligible New ART Patients Initiated on IPT; (b) Percentage of ART Patients With a Viral Load Test Conducted; (c) Percentage of PHC Clinic Attendees Screened for TB; (d) Percentage of HIV Target Achieved; (e) Percentage of HIV-TB Coinfected Patients Initiated on ART
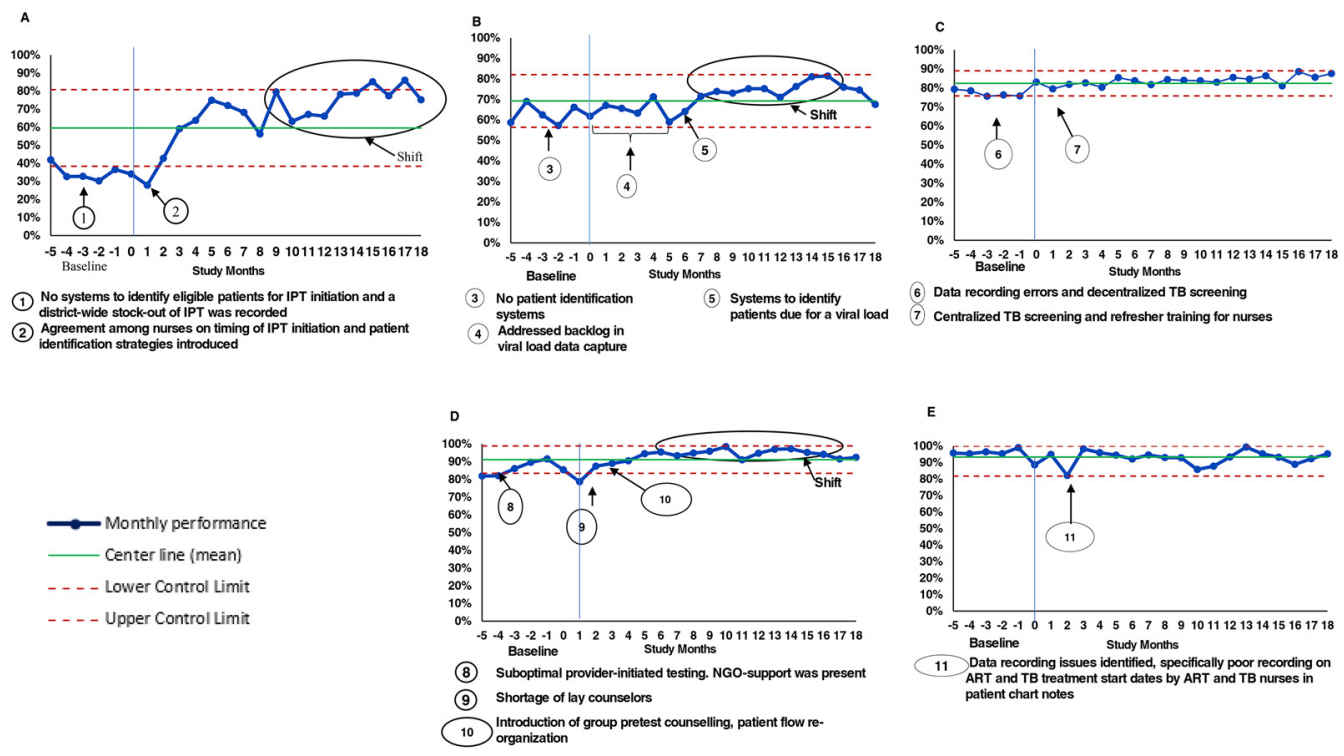

Abbreviations: ART, antiretroviral therapy; IPT, isoniazid preventive therapy; PHC, primary health care.

baseline and post QI intervention phases were statistically significant for each indicator. Completed PDSA templates were examined by 2 study staff members and common systems weaknesses and associated change-ideas were identified and summarized.

\section{Ethics}

The SUTHI trial was approved by the Biomedical Research Ethics Committee of the University of KwaZulu-Natal (BREF Ref 108/14). Informed consent for the study was waived. 
The KwaZulu-Natal Health Research and Knowledge Management committee granted permission to access PHC clinics in the study districts of KwaZulu-Natal (HRKM309/14).

\section{RESULTS}

The QI intervention was conducted from December 1, 2016, to January 1, 2019. Table 3 provides a summary of health care workers who attended the 3 learning sessions. At no learning session were all 8 PHC clinic supervisors present.

Clinic QI teams identified HIV-TB processes for improvement based on findings of patient- and work-flow analyses and suboptimal performance at baseline (Table 4). Systems weaknesses and opportunities for improvement were identified in all clinics for IPT initiation and VL testing at month 12 after ART initiation. However, HTS, TB screening, and ART initiation became the foci of QI initiatives in, 17 and 3 clinics, respectively. Clinics that did not actively engage in improving an indicator continued to monitor performance only. All clinics were included in analyses of the performance of the collaborative.

IPT initiation at baseline was 15.9\% (95\% confidence interval $(\mathrm{CI})=4.8,52.5) \quad($ Table 2$)$. The main causes of poor IPT initiation were identified as uncertainty among nurses on timing of IPT initiation in new ART patients and weak systems to identify returning ART patients who were eligible for IPT (Table 4). The improvement in IPT initiation observed after the start of the QI intervention was due to a district-level IPT stock-out in the baseline period. (Figure 3A). By study month 6, a $64.8 \%$ IPT initiation rate was achieved. In the last 6 months of the study, the QI collaborative achieved a mean of $76.4 \%(95 \% \mathrm{CI}=66.3,88.1)$, a 5 -fold higher mean than at the baseline phase, $P=.019$ (Table 2). On average clinics carried out 4 PDSA cycles to improve IPT initiation, and while major improvement was observed, the target of $90 \%$ was never attained in the study. Improvement in IPT performance is observed from month 1; however, a shift above the mean was observed from month 9 to 18 (Figure 3A).

At baseline, the mean rate of VL testing was $61.4 \% \quad(95 \% \quad \mathrm{CI}=56.4,66.8), 28.6 \%$ below the $90 \%$ desired target. Major backlogs in VL data entry that generated inaccurate VL completion reports were the main cause of poor performance identified by the QI teams. In the first 6 months post-study enrollment, no QI activities were recorded in any QI clinics to improve VL, instead, efforts to reduce the data entry backlog for the last 12 months were undertaken and QI activities were started closer to study month 6 (Figure 3B). A shift above the mean was observed from month 6 to 16. During the last 6 months of the study, the

TABLE 3. District and Clinic Staff Trained in Quality Improvement Methods for a Quality Improvement Intervention for HIV/TB Service Integration in KwaZulu-Natal, South Africa

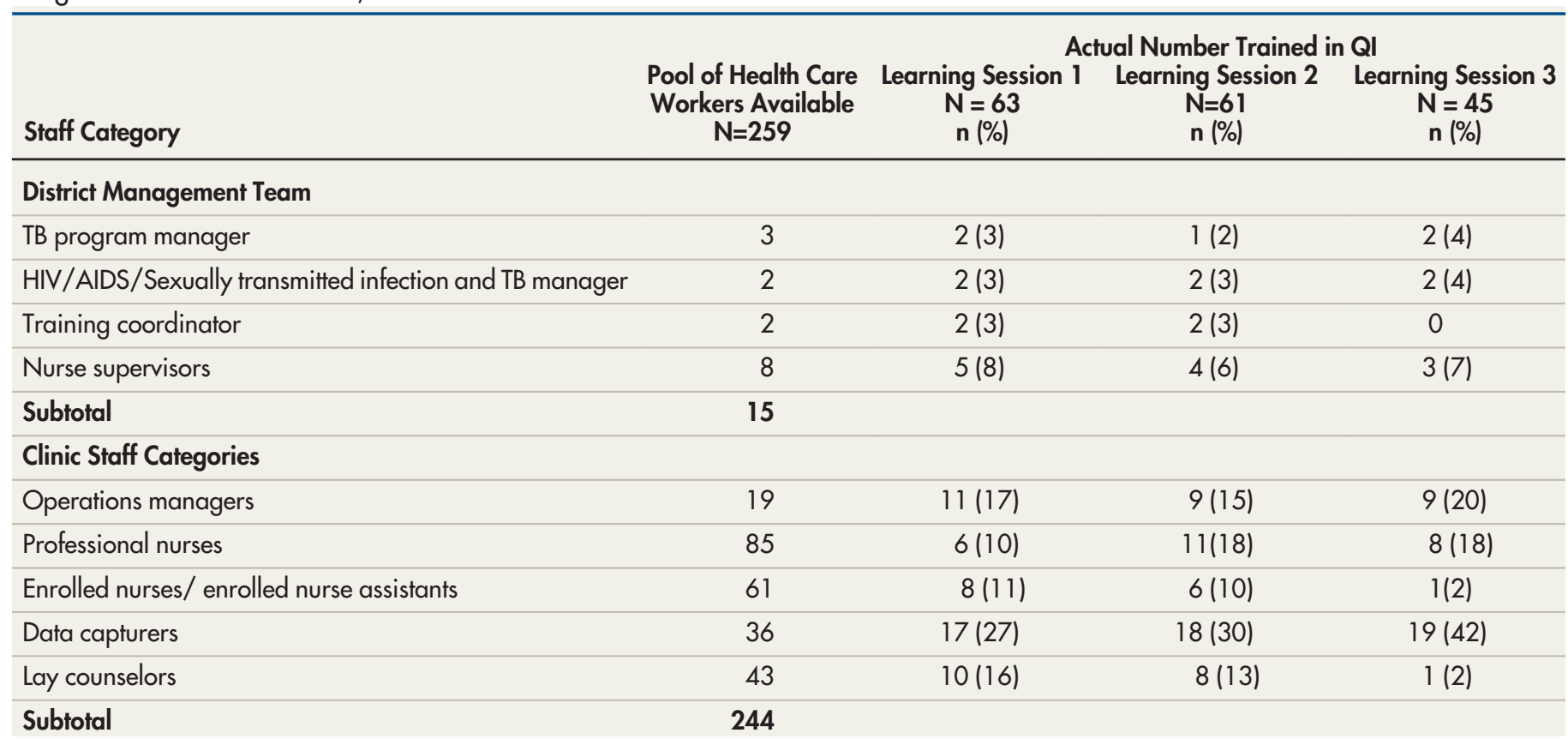


TABLE 4. Health Systems Weaknesses Identified and Associated Change Ideas for a Quality Improvement Intervention for HIV/TB Service Integration in KwaZulu-Natal, South Africa

HIV-TB Process Health Systems' Weaknesses Identified Change Concepts

HTS Relying only on patient requests or referrals for HIV testing.

- Acute patients were overlooked for HTS services (e.g., wound care patients)

HTS data inaccuracies caused by:

- Not completing HTS registers in real-time

- Misplacing HTS registers

Overdependence on lay counselors

- HTS viewed as the work of lay counselors

- Lack of counseling skills among nurses to relieve/stand-in for lay counselors
Introduced strategies to enhance provider-initiated testing:

- Offered group pretest counseling in all patients' waiting areas

- Implemented a daily roster system of staff to conduct pre-test counseling

- Nurse in charge or designee to check accountability log daily

Redesigned clinic patient flow

- Ensure that acute patients are directed to lay counselors after vitals assessments ${ }^{a}$ are conducted

- If above not possible, then staff caring for acute patients were (i) trained in HIV testing and counseling and (ii) provided with the appropriate HTS stationery

Daily data quality control checks

- Daily quality control of HTS registers and frequent audits of patient files and electronic data to ensure HIV status is known for all patients

\section{Increasing the accountability and responsibility for the HTS}

program

- On-site HTS refresher training was held which addressed: preand post-test counseling messages, conducting HIV rapid tests, and data recording

- Awareness of clinic target set by the district health office was disseminated

\section{TB screening among Missed opportunities to offer TB screening to all} PHC clinic attendees clinic attendees

\section{Centralized TB screening}

- Made TB screening mandatory at an identified strategic point visited by all patients, such as, vitals assessment ${ }^{a}$ station

- Visual prompts and reminders to conduct TB screening included large and colorful TB posters, printed and easily accessible signs, and symptoms checklists

- Made TB screening mandatory for acute patients

Inaccurate TB screening data

Data quality control checks
- Daily data quality control checks conducted by nurse in charge or designee to check:

Completeness and accuracy of daily TB screening register

Number of symptomatic patients and number of sputum samples sent for Xpert/ MTB Rif ${ }^{b}$ testing

- Quality control of clinic headcount ${ }^{c}$ data:

- Exclude patient representatives or family members Subtract TB confirmed patients from the clinic headcount Mass TB screening campaigns conducted in communities must be distinguishable from screening conducted in the clinic

\section{Clarify IPT initiation timing and arrive at mutually agreed upon} timing for initiation

- Each clinic team arrived at a common time to start IPT (e.g. 7,14 , or 30 days after starting ART)

- Agreed upon timing was documented and standardized for entire clinic 
TABLE 4 Continued

HIV-TB Process Health Systems' Weaknesses Identified Change Concepts

Confusion about roles and responsibilities of clinic staff

No system for identifying patients eligible for IPT
Enhancing accountability and responsibility for IPT program

- Roles and responsibilities were assigned to all staff categories and documented

Strategies to identify patients returning at the agreed upon time for IPT

- The "box system" -eligible patients' files placed in a decorated box for easy identification, OR

- Tagged files of eligible patients with stickers or red ink OR

- The "diary system" reminder note in clinic diary to initiate IPT at next visit and note attached to patient file

Poor recording of IPT initiation date in clinic chart notes

Refresher training on clinic stationery to document IPT

- Nurses directed to document start date in designated fields and data capturers shown where to find the start date

Nurses lack confidence to rule out TB

Host a training for nurses, lay counselors, and data capturers

highlighting the importance and potential benefit of IPT for HIVinfected patients

- Link this training with the TB screening training (above) to boost confidence to rule out TB
ART initiation among HIV-TB coinfected patients

\author{
Patient chart notes for TB and ART kept \\ separately \\ - TB and ART files not integrated
}

- No unique identifier for TB and ART file nurses regarding ART and TB treatment initiation
Poor coordination between NIMART and TB

\section{Combining ART and TB files} physically combined

- The district health office agreed upon a common unique identifier to be used

- The TB module on the electronic ART database was activated to accommodate TB and ART data

\section{Refresher training for nurses}

- Clarified patient flow for ART initiation visits in TB/HIV coinfected patients

- Improved chart notes for ART and TB treatment start dates
- For HIV-TB coinfected patients, ART and TB chart notes were

$\begin{array}{ll}\text { VL monitoring at } & \text { No system to identify patients eligible for } \\ \text { month } 12 \text { after ART } & \text { month } 12 \text { VL tests } \\ \text { initiation } & \end{array}$

Generate report from electronic system of patients due for VL

- Address the data capturing backlog of VL results and ART initiation

- Draw on the assistance of local nongovernmental organizations and support partners for assistance with data capture

- Generate VL reports from the data system to determine which patients have not had or are due for VL test (filter out deceased and transferred-out patients)

- Tag/mark the files of patients due for VL for easy identification

- Trace patients who were missed for a VL test

Abbreviations: ART, antiretroviral therapy; HTS, HIV testing services; IPT, isoniazid preventive therapy; NIMART, Nurse Initiated Management of Antiretroviral Therapy; PHC, primary health care; VL, viral load.

a Vitals assessments refers to general measures of well-being which typically include weight, body temperature, blood pressure measurements.

${ }^{b}$ Xpert/MTB RIF a rapid, molecular, cartridge-based test used for TB diagnostics that provides an immediate rifampicin resistance result.

${ }^{c}$ Clinic headcount refers to the total number of patients who accessed the clinic for any type of clinical service.

mean VL monitoring rate was $12.6 \%$ higher than the baseline rate $(P=.045)$, which was less than $50 \%$ of what was needed to meet the target (Table 2).
Data inaccuracies were noted at baseline for TB screening (Figure 3C). Data quality checks and refresher training were change ideas tested for improvement (Table 4). Mean TB screening rates 
improved by $9 \%$ between the baseline period and post-QI intervention (Table 2), and the 90\% target was not achieved by the collaborative.

Mean ART initiation rates were greater than $90 \%$ at baseline and continued post-intervention period (Table 2). The monthly performance in ART initiation was addressed only in 3 clinics, and the decrease of $1.7 \%$ was not significantly different from baseline performance $(P=.481)$. HTS was the only indicator that was improved and exceeded the $90 \%$ target (Figure 3D, Table 2).

\section{DISCUSSION}

This article describes the QI intervention implemented in the SUTHI cluster-randomized trial to improve HIV-TB health care performance. In South Africa, integrated HIV-TB services are mandatory, and this study shows that improvement in HIV-TB process outcomes is needed and possible. Using the Model for Improvement, we showed that IPT initiation improved substantially; whereas HIV testing, TB screening, and VL monitoring were moderately improved, and ART initiation among HIV-TB coinfected patients was an already well-performing indicator that required monitoring and only a few clinics had to strengthen coordination between the TB nurses and ART-initiating nurses. An important output of the QI intervention was a set of change ideas that are potentially transferrable to other settings and could contribute to the improvement of integrated HIV-TB services.

Several factors can be attributed to the success of IPT initiation rates in this study. First, clarifying nonspecific initiation guidelines improved decisiveness among nurses in the timing of IPT initiation. Second, as IPT is an indicator monitored at the district and provincial levels, clinic staff were motivated to improve IPT performance. Third, low performance at baseline $(15.9 \%)$, increased the likelihood and potential for improvement. Fourth, improving IPT initiation and data completeness in patient files and on IPT dispensing and stock charts, subsequently improved the IPT supply chain. The supply of IPT depends on demand for IPT. Improved IPT dispensing data provided a better reflection of the clinics' demand for IPT, and the ordering of stock was adjusted accordingly. Interestingly, approximately 6 months of QI to improve IPT and HTS was undertaken before the shift was observed. This may indicate that clinics require approximately 6 months to completely embed new processes into the clinic.

Three systematic reviews evaluating the effectiveness of QI collaboratives concurred that the size of improvement observed is often a function of baseline performance and low-performing indicators are more likely to have larger improvement. $^{23,24}$ A QI approach to improving IPT initiation was successful in other resourceconstrained settings. In a Namibian case study of QI capacity development, IPT initiation resulted in a $12 \%$ increase (from $16 \%$ to $28 \%$ ) at a national level. ${ }^{25}$ In a Nigerian case study, situated at a single state-run hospital, IPT initiation improved by $39 \%(11 \%$ to $50 \%){ }^{26}$ Interestingly, the Namibian study was at a national level and the Nigerian study was conducted at 1 facility. ${ }^{25,26}$ Similar to the SUTHI study, the Nigerian study was more active in addressing issues of organization, process, management, and systems. The authors surmise that root cause analysis and firsthand involvement of clinic staff in developing systems played a role in achieving improvement. ${ }^{26}$

A systematic review of strategies to improve health care performance showed that large improvements (defined as 20-30 percentage point improvement) are generally achieved in strategies that used a combination of training, collaborative learning, supervision, and improvement of infrastructure (such as data quality improvement), as was done in the SUTHI trial. ${ }^{27}$ Yet, providerinitiated HIV testing and TB screening achieved modest improvement (defined as 5-10 percentage points). VL monitoring moderately improved from baseline (defined as between 10-20 percentage points) and ART initiation slightly decreased. These results are evidence that other factors drive the success of an improvement strategy. The role of contextual factors in influencing improvement outcomes is emerging as an important consideration when assessing QI initiatives. ${ }^{28,29}$ Work culture, access to knowledge resources, QI leadership, supportiveness of work environments, and staff motivation and willingness to question the status quo, are but a few examples of contextual factors that may influence the success of QI initiatives. ${ }^{28,30,31}$

\section{Lessons Learned}

In the SUTHI trial, we identified important factors that may explain the suboptimal improvement for some indicators. The effect of baseline performance was to the advantage of IPT improvement; however, HIV testing services and ART initiation in HIVTB coinfected patients were high at baseline, and there was little room for improvement thereafter. Future QI interventions should consider baseline performance when setting expectations for improvement, however, we do not recommend that 
baseline performance be considered the sole criteria for selecting indicators for improvement. This study showed that there are indicators that are close to reaching targets but appear to be plateauing, for example, VL testing (Figure 3B) and TB screening (Figure 3C). QI improved both indicators and still has a role to play in addressing the barriers that prevent these indicators from reaching the desired target of $90 \%$.

Capacitating clinic staff with data analytic skills is an important factor in ensuring the success of QI interventions because it improves technical skills, confidence, and self-efficacy of clinic teams. ${ }^{32}$ In addition, monitoring improvement using routine data is fundamental to the QI intervention. Learning sessions covered the basics of how routine data can be analyzed (e.g., calculation of percentages, means, and medians), plotted onto run charts, and interpreted using run-chart rules. QI mentors reinforced this knowledge at QI mentorship visits. Poor data quality threatens clinic teams' efforts to monitor improvement and is a barrier to successful QI implementation. ${ }^{33}$ Despite our attempts to address the completeness and accuracy of routine data, poor data quality undermined our QI intervention. For example, TB screening data were adversely impacted by inflated clinic headcount numbers (the denominator), incorrect completion of TB screening registers, and misplaced TB registers. The success of VL monitoring improvement depended on accurate and complete data entered into the patient electronic database; however, nearly 6 months of addressing data entry backlogs reduced the time available to improve the indicator coupled with challenges of tracing of patients to return to the clinic. Tracking patients is a resource-intensive effort due to poor telephonic services, lack of vehicles, and incomplete patient contact information (namely, telephone/mobile data, lack of street addresses).

A Ugandan-based QI project that aimed to improve TB case notification also relied on routine data to monitor improvement and went beyond checking clinic registers for completeness and accuracy. ${ }^{34}$ A data tool was used to triangulate patient data from multiple sources, that is, laboratory data, patients' chart notes, and TB laboratory data. ${ }^{34}$ Unfortunately, no data metrics were available to quantify the extent to which data was improved. A South African QI project to prevent mother-to-child transmission of HIV in labor wards used a specially designed checklist that included prompts for nurses to complete and document important tasks. ${ }^{17}$ Following this intervention, there was a marked improvement in data quality with erroneous data, namely, percentages greater than $100 \%$ being eliminated. These studies demonstrate that innovative measures are needed to improve the quality of routine data and adding additional human resources to improve the completeness and accuracy of data may not be sufficient.

We intended for PHC clinic supervisors to lead the QI intervention, but their involvement was limited by their workloads and conflicting meetings. Clinic staff selected to attend the learning sessions did not always pass on their learnings from the workshops to their colleagues and the studyappointed nurse mentors reported resistance from non-workshop attendees to the workflow changes. A mixed-methods study identified personal- and work-environment-related factors that influence a health care worker's ability to transfer knowledge from QI trainings to peers. ${ }^{35}$ Health care workers that are successful in transferring training knowledge have a positive attitude to implementing changes, interpersonal skills to address resistance from peers, and the ability to question the status quo. ${ }^{35}$ A work environment in which teams are receptive to new ideas, supportive of change, and leadership support is present, facilitates the transfer of training knowledge. ${ }^{35}$ In the SUTHI trial, selection of clinic staff to attend learning sessions was at the discretion of the PHC clinic nurse supervisor and nurse in charge of the clinic. While individuals from all clinic departments were trained, staff categories, such as data capturers and lay counselors may not have been empowered enough to transfer their new knowledge to more senior colleagues. Future QI interventions must consider screening potential QI trainees for the appropriate qualities that will allow for the transfer of QI knowledge.

\section{Challenges in QI Implementation}

Implementation of QI at the clinic level was accompanied by several challenges. First, QI was vaguely understood in both districts and clinic teams often believed that they were implementing QI by virtue of the weekly nerve center meetings and discussing problems and challenges at staff meetings. The need for the SUTHI QI intervention was initially unclear to QI clinics. The learning sessions established the importance of using a QI approach that is guided by a framework (Model for Improvement and PDSA), uses tools (e.g., process charts), locally developed strategies (change ideas), and monitoring progress with

\section{Despite our attempts to address the completeness and accuracy of routine data, poor data quality undermined our QI intervention.}

\section{Future QI interventions must consider screening potential QI trainees for the appropriate qualities that will allow for the transfer of QI knowledge.}




\section{Consistent visits and mentorship by the QI nurse mentors were critical in demonstrating how the frameworks and tools translated to practice.}

data. Importantly, the consistent visits and mentorship by the QI nurse mentors were critical in demonstrating how the frameworks and tools translated to practice.

Secondly, QI implementation adds additional work for clinic staff, in that data needed to be collected and recorded to track progress more frequently. While change ideas were implemented, it was a challenge to keep staff motivated to track their performance. For example, in HTS, group pretest counseling was a key change idea; however, the source documents developed to track the number of group pretest counseling sessions in patients' waiting areas were not completed.

Third, leadership at the clinic level was supportive of the QI intervention; however, due to many commitments in and outside of the clinic, there was little involvement of clinic leaders in the QI meetings. This delayed implementation of some change ideas, as junior-level clinic staff do not have major decision-making power to make changes, such as in clinic patient flow.

\section{Limitations}

The study had limitations. First, while the QI intervention was implemented in the context of a randomized controlled trial, we were unable to prevent exposure of QI clinics from other improvement interventions to enhance integrated HIV-TB services, particularly, improvement efforts of the DMTs and technical assistance from local NGOs. Motivated DMTs in both study districts frequently monitored the progress of HIV and TB process indicators, fed back to poorly performing clinics, and conducted quarterly in-person visits to all clinics. The true effect of the QI intervention has likely been masked by the improvement efforts of the DMTs and local NGOs. Although the study was unable to separate the effect of the DMTs' efforts and QI intervention efforts on improvements observed, the baseline period (Figure 3A-3E) offers some insight into the performance before and after the QI intervention was implemented. The QI intervention ideally complemented the performance monitoring and feedback efforts of the DMTs which were seldom able to conduct in-depth root cause analyses of systems weaknesses and develop clinic-specific interventions. Second, the study was not adequately resourced to determine if improvements in the QI clinics were sustained beyond the study period or if the QI tools, strategies, and best practices were scaled up to more clinics in other areas. Staff turnover and changes in key personnel, who were trained in QI methods, may add to the challenge of sustaining and scaling up QI activities once the study ended. Third, as per the study design, all analyses were at the cluster level and clinics within each cluster were considered as 1 unit. However, the QI intervention was at the clinic level, and different clinics within a cluster adopted different change ideas (such as the different timing of IPT initiations in Table 4), and we could not compare clinics to determine which change ideas translated to larger improvements.

\section{CONCLUSION}

This study showed that a QI approach to improving HIV-TB health care delivery is feasible and uptake of QI among clinic teams is evident across all indicators. With guidance, clinic staff can reveal weaknesses and gaps known only to the people who work within a system. Baseline performance of an indicator should be considered when setting expectations and assessing the size of improvement. Efforts to improve the quality of routine HIV and TB data need to be intensified for future QI efforts to be successful. The importance of basic clinical skills training should not be underestimated; however, innovative approaches to teaching health care workers need to be introduced for information to be retained and facilitate practical application. Lastly, QI complements the efforts of local NGOs and routine monitoring activities of the South Africa DOH.

Acknowledgments: We thank the South African Department of Health, District Management Teams of the Ugu and King Cetshwayo Districts for supporting this study; the nurse supervisors and clinic staff for sharing their best practices, supporting the study, and collaboration between clinics; the CAPRISA SUTHI Quality Improvement nurse mentors and the roving Data Management Team for their tireless efforts in the field.

Funding: SG is currently a University of KwaZulu-Natal (UKZN) Developing Research Innovation, Localisation and Leadership in South Africa (DRILL) fellow. DRILL is a NIH D43 grant (D43TW010131) awarded to UKZN in 2015 to support a research training and induction program for early-career academics. SG was supported by the European and Developing Countries Clinical Trials Partnership (EDCTP) Grant TMA2018SF-2467. The research reported in this manuscript was supported by the South African Medical Research Council, with funds received from the South African National Department of Health, and the United Kingdom (UK) Medical Research Council, with funds received from the government's Newton Fund. This UK-funded award is part of the EDCTP2 program supported by the European Union.

Disclaimer: The content is solely the responsibility of the authors and does not necessarily represent the official views of Developing Research Innovation, Localisation and Leadership and the National Institutes of Health. The funders had no role in the study design, execution of the study, analyses and interpretation of data, or the decision to submit results for publication.

Author contributions: SG was responsible for the study conduct, had oversight of the study operations, development of the first draft of the manuscript, intellectual input, analysis, and interpretation of the results. KN edited and reviewed the manuscript and had oversight of the study. RM led the field team and edited the manuscript. MFT provided guidance to the field 
team on Ql implementation and interpretation of results. AJN and NYZ provided input on the analysis and interpretation of results. NP provided intellectual input and contributed to the writing of the manuscript. MT edited the manuscript and provided intellectual input. PMB provided input on the study design and manuscript. ML provided input on the interpretation of the data, intellectual input, and editing of the manuscript.

Competing interests: None declared.

\section{REFERENCES}

1. Country factsheets: South Africa 2020. Joint United Nations Programme on HIV/AIDS. Accessed August 18, 2021. https:// www.unaids.org/en/regionscountries/countries/southafrica\#: : text $=\ln \% 20$ South\%20Africa\%20in\%202018,49\%20years)\%20was $\% 2020.4 \% 25$

2. World Health Organization (WHO). Global Tuberculosis Report 2020. WHO; 2020. Accessed August 22, 2021. Accessed August 23, 2021. https://apps.who.int/iris/rest/bitstreams/1312164/ retrieve

3. Naidoo P, Theron G, Rangaka MX, et al. The South African tuberculosis care cascade: estimated losses and methodological challenges. J Infect Dis. 2017;216(suppl_7):S702-S13. CrossRef. Medline

4. World Health Organization (WHO). WHO Policy on Collaborative TB/HIV Activities. Guidelines for National Programmes and Other Stakeholders. WHO; 2012. Accessed August 18, 2021. http:// apps.who.int/iris/bitstream/handle/10665/44789/ 9789241503006_eng.pdf

5. Legido-Quigley $\mathrm{H}$, Montgomery $\mathrm{CM}$, Khan $\mathrm{P}$, et al. Integrating tuberculosis and HIV services in low- and middle-income countries: a systematic review. Trop Med Int Health. 2013;18(2):199-21 1. CrossRef. Medline

6. Scott VE, Sanders D. Evaluation of how integrated HIV and TB programs are implemented in South Africa and the implications for rural-urban equity. Rural Remote Health. 2013;13(2):2165. Medline

7. Naidoo K, Gengiah S, Yende-Zuma N, et al. Addressing challenges in scaling up TB and HIV treatment integration in rural primary healthcare clinics in South Africa (SUTHI): a cluster randomized controlled trial protocol. Implement Sci. 2017;12(1):129. CrossRef. Medline

8. South African National Department of Health (NDOH). National Consolidated Guidelines: For the Management of HIV in Adults, Adolescents, Children and Infants and Prevention of Mother-to-Child Transmission. NDOH, 2020. Accessed August 23, 2021. https:// www.knowledgehub.org.za/system/files/elibdownloads/2020 07/National\%20Consolidated\%20Guidelines\%2030062020\% 20signed\%20PRINT\%20v7.pdf

9. Chihota VN, Ginindza S, McCarthy K, Grant AD, Churchyard G, Fielding K. Missed opportunities for TB investigation in primary care clinics in South Africa: experience from the XTEND Trial. PLoS One. 2015;10(9):e0138149. CrossRef. Medline

10. Kweza PF, Van Schalkwyk C, Abraham N, Uys M, Claassens MM Medina-Marino A. Estimating the magnitude of pulmonary tuberculosis patients missed by primary health care clinics in South Africa. Int J Tuberc Lung Dis. 2018;22(3):264-272. CrossRef. Medline

11. Padayatchi N, Daftary A, Naidu N, Naidoo K, Pai M. Tuberculosis: treatment failure, or failure to treat? Lessons from India and South Africa. BMU Glob Health. 2019;4(1):e001097. CrossRef. Medline

12. Loveday M, Zweigenthal V. TB and HIV integration: obstacles and possible solutions to implementation in South Africa. Trop Med Int Health. $2011 ; 16(4): 431-438$. CrossRef. Medline

13. IHI partners with South African National Department of Health on initiative to improve Tuberculosis care and outcomes. Press release. Institute for Healthcare Improvement; August 17, 2017. hHtp://www. ihi.org/about/news/Documents/IHIPressRelease_GatesFoundation-Funds-TB-Work-in-South-Africa_Aug17.pdf
14. Reid A, Abraczinskas M, Scott V, et al. Using collaborative coalition processes to advance community health, well-being, and equity: a multiple-case study analysis from a national community transformation initiative. Health Educ Behav. 2019;46(1_suppl):100S-09S CrossRef. Medline

15. Berwick DM. The science of improvement. JAMA. 2008;299 (10):1182-1184. CrossRef. Medline

16. Mate KS, Ngubane G, Barker PM. A quality improvement model for the rapid scale-up of a program to prevent mother-to-child HIV transmission in South Africa. Int J Qual Health Care. 2013;25 (4):373-380. CrossRef. Medline

17. Youngleson MS, Nkurunziza P, Jennings K, Arendse J, Mate KS, Barker P. Improving a mother to child HIV transmission programme through health system redesign: quality improvement, protocol adjustment and resource addition. PLoS One. 2010:5(11):e13891. CrossRef. Medline

18. Massyn N, Peer N, English R, Padarath A, Barron P, Day C, eds District Health Barometer 2015/2016. Health Systems Trust; 2016. Accessed August 18, 2021. https://www.hst.org.za/publications/ District\%20Health\%20Barometers/District\%20Health\%20Barometer \%202015_16.pdf

19. Uyei J, Coetzee D, Macinko J, Weinberg SL, Guttmacher S. Measuring the degree of integrated tuberculosis and HIV service delivery in Cape Town, South Africa. Health Policy Plan. 2014;29 (1):42-55. CrossRef. Medline

20. Joint United Nations Programme on HIV/AIDS (UNAIDS). 90-9090: An Ambitious Treatment Target to Help End the AIDS Epidemic. UNAIDS; 2014. Accessed August 18, 2021. https://www.unaids. $\mathrm{org} /$ sites/default/files/media_asset/90-90-90_en.pdf

21. The Breakthrough Series: IHI's Collaborative Model for Achieving Breakthrough Improvement. Institue for Healthcare Improvement (IHI) Innovation Series white paper. IHI; 2003. Accessed August 18, 2021. http://www.ihi.org/resources/Pages/IHIWhitePapers/ TheBreakthroughSerieslHIsCollaborativeModelforAchieving Breakthroughlmprovement.aspx

22. Lee K, McGreevey C. Using control charts to assess performance measurement data. Jt Comm J Qual Improv. 2002;28(2):90-101. CrossRef. Medline

23. Franco LM, Marquez L. Effectiveness of collaborative improvement: evidence from 27 applications in 12 less-developed and middleincome countries. BMJ Qual Saf. 2011;20(8):658-665. CrossRef. Medline

24. Irwin R, Stokes T, Marshall T. Practice-level quality improvement interventions in primary care: a review of systematic reviews. Prim Health Care Res Dev. 2015;16(06):556-577. CrossRef. Medline

25. Bardfield J, Agins B, Akiyama M, et al. A quality improvement approach to capacity building in low- and middle-income countries. AIDS. 2015;29 Suppl 2:S179-S186. CrossRef. Medline

26. Ogunsola $\bigcirc \mathrm{O}$, Ajayi $\bigcirc, \mathrm{O}_{\mathrm{O}} \mathrm{O}$, et al. Improving coverage and completion rate of isoniazid preventive therapy among eligible HIV patients using quality improvement approaches: a case study of State Hospital, liebu Ode, Ogun State, Nigeria. Pan Afr Med J. 2019;34:193. CrossRef. Medline

27. Rowe SY, Peters DH, Holloway KA, Chalker J, Ross-Degnan D, Rowe AK. A systematic review of the effectiveness of strategies to improve health care provider performance in low- and middle-income countries: methods and descriptive results. PLoS One. 2019;14(5): e0217617. CrossRef. Medline

28. Li SA, Jeffs L, Barwick M, Stevens B. Organizational contextual features that influence the implementation of evidence-based practices across healthcare settings: a systematic integrative review. Syst Rev. 2018;7(1):72. CrossRef. Medline

29. Rowe AK, Rowe SY, Peters DH, Holloway KA, Chalker J, Ross-Degnan $D$. Effectiveness of strategies to improve health-care provider practices in 
low-income and middle-income countries: a systematic review. Lancet Glob Health. 2018;6(11):el 163-e1 175. CrossRef. Medline

30. Bergström A, Skeen S, Duc DM, et al. Health system context and implementation of evidence-based practices-development and validation of the Context Assessment for Community Health (COACH) tool for low- and middle-income settings. Implement Sci. 2015;10:120. CrossRef. Medline

31. Kaplan HC, Brady PW, Dritz MC, et al. The influence of context on quality improvement success in health care: a systematic review of the literature. Milbank Q. 2010;88(4):500-559. CrossRef. Medline

32. Shea CM, Turner K, Albritton J, Reiter KL. Contextual factors that influence quality improvement implementation in primary care: the role of organizations, teams, and individuals. Health Care Manage Rev. 2018;43(3):261-269. CrossRef. Medline

33. Rendell N, Lokuge K, Rosewell A, Field E. Factors that influence data use to improve health service delivery in low- and middle-income countries. Glob Health Sci Pract. 2020;8(3):566-581. CrossRef. Medline

34. Karamagi E, Sensalire S, Muhire M, et al. Improving TB case notification in northern Uganda: evidence of a quality improvementguided active case finding intervention. BMC Health Serv Res. 2018;18(1):954. CrossRef. Medline

35. Eid A, Quinn D. Factors predicting training transfer in health professionals participating in quality improvement educational interventions. BMC Med Educ. 2017;17(1):26. CrossRef. Medline

Peer Reviewed

Received: February 9, 2021; Accepted: July 22, 2021.

Cite this article as: Gengiah S, Naidoo K, Mlobeli R, et al. A quality improvement intervention to inform scale-up of integrated HIV-TB services: lessons learned from KwaZulu-Natal, South Africa. Glob Health Sci Pract. 2021;9(3):444-458. https://doi.org/10.9745/GHSP-D-21-00157

(C) Gengiah et al. This is an open-access article distributed under the terms of the Creative Commons Attribution 4.0 International License (CC BY 4.0) which permits unrestricted use, distribution, and reproduction in any medium, provided the original author and source are properly cited. To view a copy of the license, visit https://creativecommons.org/licenses/by/4.0/. When linking to this article, please use the following permanent link: https:// doi.org/10.9745/GHSP-D-21-00157 